\title{
Compound cantharis revers chemotherapy resistance of TNBC via miR-520d/Beclin-1 signal axis
}

\author{
Hongchang $\mathrm{Li}^{1 *}$, Huan Liu ${ }^{2 *}$, Yafeng Chen ${ }^{2}$, Jie Li ${ }^{2}$, Weiyan Liu ${ }^{1}, \mathrm{Ke} \mathrm{Xu}^{3 *}$, Dianxu Feng ${ }^{2 *}$ and Xia Sheng ${ }^{4 *}$ \\ ${ }^{1}$ Department of General Surgery, Institute of Fudan-Minhang Academic Health System, Minhang Hospital, Fudan University, China \\ ${ }^{2}$ Department of General Surgery, Putuo Hospital, Shanghai University of Traditional Chinese Medicine, China \\ ${ }^{3}$ Central laboratory, Putuo Hospital, Shanghai University of Traditional Chinese Medicine, China \\ ${ }^{4}$ Department of Pathology, Institute of Fudan-Minhang Academic Health System, Minhang Hospital, Fudan University, China \\ \#These authors Contributed to the study equally
}

\begin{abstract}
Context: In vitro and in vivo research has shown that compound cantharis (CCI) can reverse chemotherapy resistance to docetaxel (Doc) of triple-negative breast cancer (TNBC).

Objective: To investigate the effect of CCI on TNBC.

Materials and methods: The human TNBC cell MDA-MB-231, MDA-MB-468 drug resistance cell model were constructed. They were divided into four groups: solvent control group (Con), Doc $(5 \mu \mathrm{mol} / \mathrm{L})$, CCI $(10 \mu \mathrm{mol} / \mathrm{L})$ and Doc + CCI. Detection of the effects of each drug on the proliferation and apoptosis of TNBC were via MTT, flow cytometry and Tunel, while on the autophagy level were through western blot (WB), MDC staining and immunofluorescence. Then observe the effect of changing the expression levels of Beclin-1 and miR-520d on the apoptosis and autophagy levels of triple-negative breast cancer cells. Two BALB/ CL nude mice drug resistance models (MDA-MB-231/Doc and MDA-MB-468/Doc) were divided into four groups ( $\mathrm{n}=7$ ): control group (Con), Doc (10mg/ $\mathrm{kg})$, CCI $(15.06 \mathrm{~g} / \mathrm{kg})$ and Doc + CCI. The drugs were administrated by caudal vein respectively every 2 days for 25 days. Proliferation and apoptosis were via Immunohistochemistry. Autophagy-related proteins and miR-520d were measured by WB and RT-PCR.

Results: Doc + CCI could further induce apoptosis of drug resistance cell and down-regulate the expression of LC3 and Beclin-1 and reduce the autophagy level ( $p<0.01)$. MiR-520d inhibited the expression of Beclin-1 and increased the sensitivity of drug-resistant cells to Doc ( $p<0.01$ ). The results of animal experiments indicated that CCI could enhance the sensitivity of TNBC cells to Doc and the inhibition of tumor cells. Doc + CCI could significantly up-regulate the expression of apoptosis protein caspase3, Bax and miR-520d, while down-regulate the autophagy related protein LC3 and Beclin-1 ( $\mathrm{p}<0.001$ ).
\end{abstract}

Conclusion: CCI might be a promissing drug for chemotherapy resistance by inhibiting the autophagy level of TNBC cells via miR-520d/Beclin-1 signal axis.

\section{Introduction}

Breast cancer is the most common cancer among women in the world, and about half a million people die of it every year [1]. Triplenegative breast cancer (TNBC) refers to a type of breast cancer that usually tests negative to the receptors of estrogen (ER), progesterone (PR) and human epidermal growth factor (HER-2) amidst accounting for $15 \%$ of all breast cancer pathological types, with poor prognosis, high recurrence, metastatic rate and mortality $[2,3]$. In view of the poor effect of endocrine therapy on TNBC, chemotherapy is considered as an important treatment [4,5]. At present, the commonly used chemotherapy drugs for breast cancer include taxane (paclitaxel, docetaxel, albumin synthesis paclitaxel) [6], anthracycline (adriamycin, epirubicin, all kinds of liposomal amamycin), anti-metabolism (capecitabine, gemcitabine) [7]. Notably, TNBC is an easy to produce chemotherapeutic drug-resistant cells that seriously affects the efficacy of breast cancer treatment options [8]. In recent years, it has been reported that the long-term application of docetaxel (Doc) can induce the abnormal increase of autophagy level of TNBC cells, which consequently induce cell resistance, while the reduction of autophagy level can enhance the sensitivity of tumor cells to Doc $[9,10]$.

miRNA is a kind of non-coding small molecule single stranded RNA with a length of 19-25 nucleotides. miRNA can degrade or inhibit the translational process of protein by complementary binding with the specific sequence of 3'-untranslated region (3'-UTR) of downstream target gene mRNA, so as to regulate the expression of target gene at the posttranscriptional level. Recently, more attention to the regulation of autophagy has been paid to the role of non-coding RNA, such as

*Correspondence to: Xia Sheng, M.D., Ph.D, Department of Pathology, Institute of Fudan-Minhang Academic Health System, Minhang Hospital, Fudan University, 170 Xinsong Road, Shanghai, China, Tel: +86 13761017906, E-mail: shengxia_021@fudan.edu.cn

Dianxu Feng, M.D., Ph.D, Department of General Surgery, Putuo Hospital, Shanghai University of Traditional Chinese Medicine, 164 Lanxi Road, Shanghai, China, Tel: +86 13636648465, E-mail: fdianxu@sohu.com

$\mathrm{Ke} \mathrm{Xu}, \mathrm{Ph} . \mathrm{D}$, Central laboratory, Putuo Hospital, Shanghai University of Traditional Chinese Medicine 164 Lanxi Road, Shanghai, China, Tel: +86 13818524869, E-mail: cola519@163.com

Key words: compound cantharis, Triple-negative breast cancer, autophagy, chemoresistance, miR-520d/Beclin-1

Received: March 02, 2021; Accepted: March 23, 2021; Published: March 26, 2021 
miRNA [11]. miRNA is usually involved in many biological processes, including proliferation, development, homeostasis, immunity, metabolism, apoptosis and cancer [12-19]. Research has shown that miRNA could affect the drug resistance of tumor cells by regulating autophagy level [20,21]. Autophagy is a kind of self-protection mechanism of cells. Usually, when the cells are under the condition of metabolic pressure, the cells will eventually degrade cell components such as cytoplasmic proteins or organelles via phagocytosis to produce nutrients and maintain cell survival [22]. However, recent studies have shown that large-scale persistent autophagy can also culminate in cell death and inhibit tumor. Autophagy, which does not only maintain the cell survival, but also contribute to cell death, has become a hot topic in cancer research and treatment [23].

Traditional Chinese Medicine (TCM) has a good effect on relieving postoperative complications of malignant tumor and reducing the side effects of radiotherapy and chemotherapy. Compound cantharis injection (CCI), one Chinese Herbal Medicine also known as Aidi injection (Z52020236, China Food and Drug Administration), the effective constituents of which are Banmao (Cantharidin), Renshen (Radix Ginseng), Huangqi (Astragaloside) and Ciwujia (Eleutherococcus senticosus). As a multi-target TCM, CCI can clear away heat and detoxify, and eliminate blood stasis. It is clinically used for the treatment of gynecological malignant tumors, primary liver cancer, lung cancer, rectal cancer, malignant lymphoma, etc [24]. CCI is also commonlyly used as a chemotherapy adjuvant for TNBC, and its main component is cantharidin $[25,26]$. An increasing number of clinical trials evaluating the role of $\mathrm{CCI}$ in chemotherapy have been reported. At present, the mechanism underlying the anti-TNBC resistant activity of compound cantharides in inhibiting autophagy has not been reported yet. Our previous studies have found that CCI can inhibit the proliferation of TNBC cells, significantly down-regulate the expression of Beclin1 protein, and reduce the level of autophagy; we further applied CCI and Doc to TNBC cells, and the results showed that the combination of the two has a stronger inhibitory effect on cell proliferation than Doc alone. In order to clarify the action mechanism of compound cantharidin on TNBC, we used miRNA microarray to detect the TNBC-resistant cells before and after treatment with CCI and found that CCI could significantly up-regulate the level of miR$520 \mathrm{~d}$, the expression difference reaches 8.3 times [27]. We also proved that miR-520d inhibited the expression of Beclin-1 by acting on the Beclin-1 3' UTR region and increased the sensitivity of drug-resistant cells to Doc [28]. This study observed the pharmacological effect of compound cantharides on the reversal of the drug resistance of TNBC. This study also sought to explore whether miRNA autophagy signal axis is the key mechanism underlying the capacity of CCI to reverse the drug resistance of TNBC.

\section{Materials and methods}

\section{Materials}

\section{Reagents and instruments}

DMEM culture medium, fetal bovine serum and trypsin were purchased from Gibco (USA). Compound cantharis injection (CCI), was bought from Guizhou Yibai Pharmaceutical Co., Ltd. Docetaxel (Doc) was supplied by MedChemExpress (USA). MTT kit was purchased from Sangon Biotech. Flow cytometry apoptosis kit and MDC Staining Kit was purchased from Jiangsu Kaiji Biotechnology Co., Ltd. Tunel kit was provided by Roche. Beclin-1, LC3 antibodies and the goat anti-rabbit second antibody marked by horseradish peroxidase (HRP) were purchased from Abcam (UK). ThermoFisher (USA) kindly provided the Trizol reagent, TaqMan miRNA reverse transcription kit, TaqMan miRNA quantitative PCR kit and Lipofectamine 2000 transfection kit. Takara $6210 \mathrm{~A}$ reverse transcription kit and realtime (RT) quantitative polymerase chain reaction (qPCR) kit were supplied by Takara Corporation (Japan). MiR-520d mimics and inhibitors were procured from Qiagen (USA). The PCR amplicon was provided by Guangzhou Ruibo Biotechnology Co., Ltd (Patent No: ZL 201010183196.4). Electrophoresis, transmembrane and gel imaging systems were bought from Bio-Rad (USA).

\section{Animals}

BALB/CL nude mice were purchased from the animal center of Shanghai slake experimental animal Co., Ltd. (License No. SCXK, 2007-0005, SPF grade), aged 3 4 weeks, weighing 16 22 g, female. The mice were fostered in the animal room of Putuo Hospital Affiliated to Shanghai University of traditional Chinese medicine under the conditions of constant temperature of $25 \sim 27^{\circ} \mathrm{C}$, constant humidity (45\% 50\%), fresh air, dust removal and bacteria removal without special pathogen (SPF grade) breeding room. All animal experiments comply with the ARRIVE guidelines.

\section{Methods}

\section{Cell culture}

MDA-MB-231 and MDA-MB-468 cell strains of human TBNC were purchased from Cell Bank of Chinese Academy of Sciences. The corresponding Doc resistant cell strains (MDA-MB-231/Doc and MDA-MB-468/Doc) were induced in our laboratory. All the cells were cultured in DMEM medium containing 10\% fetal bovine serum at 37区 in $5 \% \mathrm{CO}_{2}$ atmospheric condition. Take logarithmic growth phase cells and culture in $10 \mathrm{nmol} / \mathrm{L}$ Doc medium for 2 3 months to construct Doc resistant cell strains. In order to maintain the drug-resistant phenotypes of MDA-MB-231/Doc and MDA-MB-468/Doc, docetaxel (final concentration of $2 \mu \mathrm{m}$ ) was added always to the culture medium of drug-resistant cells, while the dosing was stopped one week prior to the experiment.

\section{Detection of cell proliferation by MTT assay}

The cells of $5 \times 10^{3}$ cell/well density were inoculated into 96 well plate. After the cells were completely attached to the wall, the corresponding drugs were added in groups and cultured in an incubator $\left(37^{\circ} \mathrm{C}, 5 \% \mathrm{CO}_{2}\right)$. After $48 \mathrm{~h}$, MTT solution $(10 \mu \mathrm{L})$ at $5 \mathrm{mg} /$ $\mathrm{mL}$ was add into each well and cultured continuously for $4 \mathrm{~h}$. After the supernatant was sucked and discarded, DMSO $(100 \mu \mathrm{L})$ was added into each well. Then, the solution was shaking for $1 \mathrm{~min}$ to fully mix the culture medium. After the reduction product of MTT was dissolved completely, the absorbance of the product was measured via enzyme scale instrument with $492 \mathrm{~nm}$ as the experimental wavelength and 630 $\mathrm{nm}$ as the reference wavelength. The half inhibitory concentration of the drug for $50 \%$ cell kill (IC50) of the drug was determined by calculating the cell survival rate.

\section{Detection of apoptosis by flow cytometry analysis}

After the target cells in the logarithmic growth stage were digested with trypsin, the cell suspension was resuspended in the complete medium and subcultured into 6-well plates. The density of each well was $1 \times 10^{6}$. On the second day, the corresponding drugs were added in groups, and then the cells were cultured for $48 \mathrm{~h}$. After that, the cells of each treatment group were collected and cleaned by phosphate buffer solution (PBS) once. The cells were suspended with 1xBinding buffer in the flow detection kit with the cell density about $2 \sim 5 \times 10^{5}$ cells/ 
$\mathrm{mL}$. Then $5 \mu \mathrm{L}$ Annexin V-FITC was added and incubated at room temperature away from light for $10 \sim 15 \mathrm{~min}$. The cells were washed with $200 \mu \mathrm{L}$ 1xBinding buffer, centrifuged at $1000 \mathrm{rpm}$ for $4 \mathrm{~min}$, and the supernatant was discarded. After that, $190 \mu \mathrm{L} 1$ xBinding buffer was used to resuspend the cells prior to the addition of propidium iodide $(10 \mu \mathrm{L})$. Finally, the result was analyzed by flow cytometry.

\section{Detection of apoptosis by Tunel}

The cells were fixed with $4 \%$ paraformaldehyde for 30 min after climbing. PBS was employed to wash the cells gently for 3 times ( $5 \mathrm{~min} /$ time). The rest operations were carried out according to Tunel apoptosis detection kit. It was worth noting that the dilution ratio of TdT Enzyme to Fluorescein-FlagEL ${ }^{\mathrm{TM}} \mathrm{TdT}$ Labeling Reaction Mix was 1:12. DAPI was used to re-stain cells at room temperature for $3 \sim 5 \mathrm{~min}$. Then the anti-quenching was used to seal the tablets. The number of apoptotic cells was observed under fluorescence microscope.

\section{Western blot for protein expression}

The proteins of each group were extracted using RIPA buffer. The BCA kit was applied to detect the total protein concentration of each group. After the protein concentration was adjusted, the equivalent protein was separated with $10 \%$ sodium dodecyl sulfate-polyacrylamide gel electrophoresis (SDS-PAGE) and transferred to polyvinylidene difluoride (PVDF) membrane by wet rotation method. Next, the PVDF membranes were blocked with $5 \%$ skimmed milk at room temperature for $1 \mathrm{~h}$. After the first antibody was added, the PVDF membranes were sealed overnight at $4^{\circ} \mathrm{C}$. On the second day, the first antibody was discarded. Then, the goat anti-rabbit second antibody marked by HRP to the PVDF membranes was added prior to sealing for $1 \mathrm{~h}$ at room temperature, followed by the dropwise addition of color developing solution to develop the color. Next, the image of protein strip was obtained by gel imaging system.

\section{Detection of autophagy by monodasylcadaverin (MDC) staining}

The cells were treated with different drugs after climbing tablets, and then cultured for $24 \mathrm{~h}$, and washed gently by PBS for $2 \sim 3$ times. The mother liquor of MDC was diluted with medium 1:10 and then added to the cells. The cells were incubated in dark for $30 \sim 45 \mathrm{~min}$, then washed with PBS for 3 times. Then, the images of autophagosomes in the cells were observed under fluorescence microscopy after the addition of an anti-fluorescence quenching tablet. And the control group (Con), CCI group and CCI + miRNA-520d inhibitor group used the same method.

\section{Detection of autophagy related protein LC3 by immunofluorescence}

Cells were cultured to a density of about $80 \%$. Cells were counted and inoculated with 6 -well plate. The inoculated density was $5 \times 10^{4}$ cells/well. The cells were cultured overnight in a cell incubator. Then the corresponding drugs were added to the cells according to the groups. The cells were cultured for $48 \mathrm{~h}$ and then centrifuged at $4^{\circ} \mathrm{C}$. The cells were fixed in $4 \%$ paraformaldehyde for $15 \mathrm{~min}$ at $4^{\circ} \mathrm{C}$, paraffin embedded and sectioned $(4 \mu \mathrm{m})$, then dewaxed with xylene and hydrated with gradient ethanol. PBS was employed to wash the cells for 3 times (3 $\mathrm{min} /$ time) at room temperature. The cells were sealed with $1 \%$ bovine serum albumin (BSA) for 30min and then BSA was removed gently. The tissue was circled as small as possible with immunohistochemistry pen, and the pre prepared first antibody (1:200) was added, and incubated overnight in $4^{\circ} \mathrm{C}$. The slices were washed with PBS for 3 times ( $3 \mathrm{~min} /$ time). Then PBS around the tissues was wiped off. Again, the tissue was circled as small as possible with immunohistochemistry pen, and the pre prepared fluorescent secondary antibody (1:100) was added, and incubated in a wet box at room temperature for $60 \mathrm{~min}$ (keep away from light). Repeated the above mentioned operation of PBS cleaning and tissue circling, and then added 4'6-diamindino-2-phenylindol (DAPI) (diluted 10 times), incubate at room temperature in dark for 3 min (no more than $5 \mathrm{~min}$ ), and then observed with confocal microscope.

\section{Detection of miRNA expression by real-time quantitative PCR}

Trizol was used to extract total RNA from cultured cells. TaqMan miRNA reverse transcription kit was then applied for reverse transcription and the relative amount of each miRNA was based on the content of U6 snRNA in the cells. PCR technique was used for amplification, while TaqMan miRNA qPCR kit or RT qPCR kit was used for quantitative analysis according to the kit instructions. All the primers used in the experiment were designed and synthesized by Guangzhou Ruibo Biotechnology Co., Ltd (Patent No: ZL 201010183196.4).

\section{Animal experiments}

After one week of adaptation, the breast cancer cell suspension with a density of $5 \times 10^{6}$ cells was implanted subcutaneously in the right armpit of nude mice. After the tumor grew to $100 \sim 200 \mathrm{~mm}^{3}$, the animals were randomly divided into four groups. According to the groups, the drugs were administrated by caudal vein respectively: $\mathrm{A}$, solvent control group (Con); B, Doc (10mg/kg); C, CCI (15.06g/kg); D, Doc + CCI. Animals were administered once every two days for 25 days. The tumor volume and the animal weight were measured every three days respectively, and the data were recorded. The calculation formula of tumor volume (TV) is: $\mathrm{TV}=1 / 2 \times \mathrm{a} \times \mathrm{b}^{2}$, where $\mathrm{a}$, $\mathrm{b}$ represent length and width respectively. Apoptosis and autophagy related proteins in the animal models was detected by WB and Immunohistochemistry. The changes of miR-520d and Beclin-1 in animal model were detected by RT-PCR.

\section{Statistical analysis}

SPSS 24.0 and GraphPad Prism 8 were applied to analyze the experimental data and plot graphs, respectively. All the experimental data were expressed as mean \pm standard deviation (SD), while the differences between groups were detected via One Way ANOVA. A statistical significance level of $p<0.05$ was accepted for this study.

\section{Results}

Detection of CCI, Doc and their combined effects on the proliferation and apoptosis of TNBC drug resistant cells

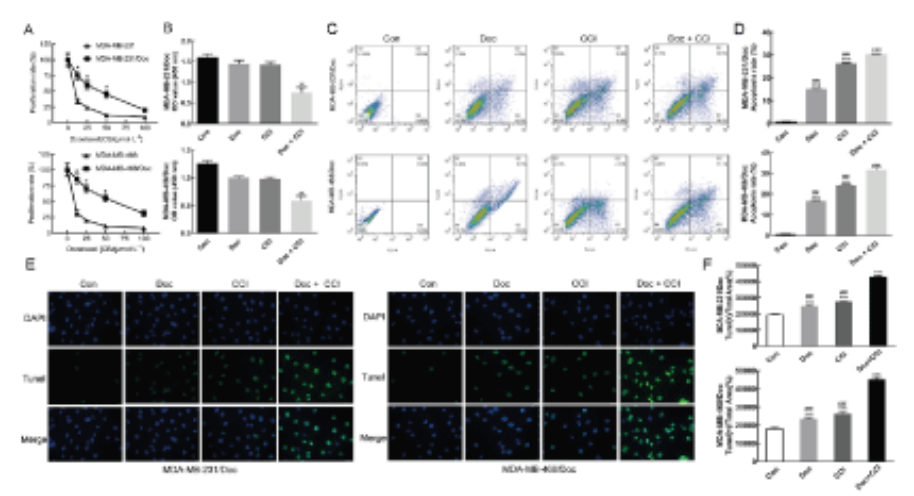

Figure 1A: Successfully constructed the drug-resistant cell MDA-MB-231/Doc and MDAMB-468/Doc; B: Effect of drugs detected by MTT on the proliferation of MDA-MB-231/ Doc and MDA-MB-468/Doc; C: Effect of drugs was detected by flow cytometry on the apoptosis of effect of on MDA-MB-231/Doc and MDA-MB-468/Doc; D: Statistics; E: Effect of drugs was detected by Tunel on the apoptosis; F: Statistics; Compared with Con, ${ }^{*} P<0.05,{ }^{* *} P<0.01,{ }^{* * *} P<0.001$; Compared with Doc + CCI, ${ }^{\prime \prime \prime \prime} P<0.001$. 
As can be seen from figure (Figure 1A), the proliferation rate of MDA-MB-231/Doc (IC50 $=38 \mu \mathrm{mol} / \mathrm{L}$ ) and MDA-MB-468/Doc (IC50 $=60 \mu \mathrm{mol} / \mathrm{L})$ cells in the drug-resistant cell group was significantly higher than that of MDA-MB-231 (IC50 = $3.8 \mu \mathrm{mol} / \mathrm{L})$, MDA-MB-468 $(\mathrm{IC} 50=6 \mu \mathrm{mol} / \mathrm{L})$. And the optical density $(\mathrm{OD})$ values of MDA-MB-231 and MDA-MB-468 decreased obviously with the increase of Doc concentration, while the OD values of MDA-MB-231/Doc and MDAMB-468/Doc did not change significantly. This result suggests that the drug-resistant cell was successfully built $(p<0.01)$. The MTT method was employed to detect the effect of the CCI, Doc and the combination of CCI and Doc on the proliferation of TNBC resistant cells. As shown in the figure (Figure 1B), the combination of Doc and CCI had synergistic effect to the enhancement of sensitivity of MDA-MB-231/Doc and MDA-MB-468/Doc to docetaxel $(p<0.01)$. The combination of drugs greatly inhibited the proliferation of drug-resistant strains than that single drug. Meanwhile, the results of flow cytometry were shown in (Figure 1C\&D). Compared with the control group, the use of CCI and DOC alone could significantly increase the cell apoptosis rate of MDAMB-231 and MDA-MB-468 drug-resistant strain (MDA-MB-231/Doc, MDA-MB-468/Doc) $(p<0.001)$. In addition, the combination of CCI and DOC further promoted the apoptosis of cells $(p<0.001)$. Tunel detection also had the same experimental results, as shown in (Figure $1 \mathrm{E})$, compared with the control group, the drug-resistant strains of Doc group and CCI group, Doc + CCI group have stronger yellow green fluorescence. Further (Figure 1F) showed that there were more apoptotic cells in MDA-MB-231/Doc and MDA-MB-468/Doc resistant strains in the combination group, indicating that the combination of DOC and CCI could further induce apoptosis $(p<0.001)$.

Detection of CCI, Doc and their combined effects on the autophagy of TNBC drug resistant cells

Western blotting showed that the level of autophagy related proteins LC3 and Beclin-1 in MDA-MB-231/Doc, MDA-MB-468/ Doc and different treatment groups were lower than that in the control group, while the level of $\mathrm{P} 62$ was higher, which suggested that the down-regulation of LC3 and Beclin-1 might be one of the main reasons for DOC and CCI to inhibit the autophagy of TNBC cells (Figure $2 \mathrm{~A} \& \mathrm{~B})$. Moreover, the combination of DOC and CCI could more
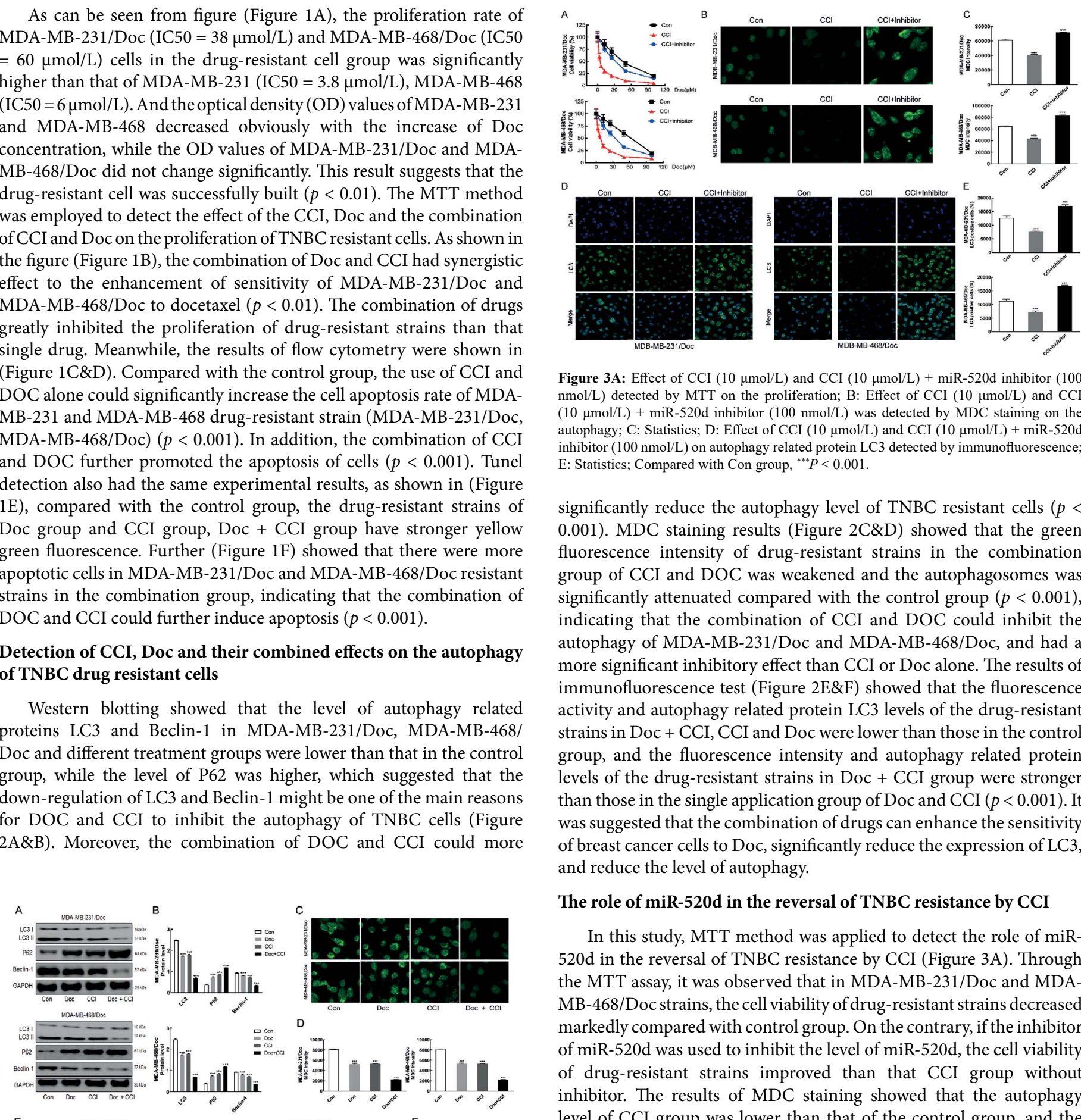

Figure 3A: Effect of CCI $(10 \mu \mathrm{mol} / \mathrm{L})$ and CCI $(10 \mu \mathrm{mol} / \mathrm{L})+\operatorname{miR}-520 \mathrm{~d}$ inhibitor $(100$ $\mathrm{nmol} / \mathrm{L})$ detected by MTT on the proliferation; B: Effect of CCI $(10 \mu \mathrm{mol} / \mathrm{L})$ and $\mathrm{CCI}$ $(10 \mu \mathrm{mol} / \mathrm{L})+\operatorname{miR}-520 \mathrm{~d}$ inhibitor $(100 \mathrm{nmol} / \mathrm{L})$ was detected by MDC staining on the autophagy; C: Statistics; D: Effect of CCI $(10 \mu \mathrm{mol} / \mathrm{L})$ and CCI $(10 \mu \mathrm{mol} / \mathrm{L})+\mathrm{miR}-520 \mathrm{~d}$ inhibitor $(100 \mathrm{nmol} / \mathrm{L})$ on autophagy related protein LC3 detected by immunofluorescence; E: Statistics; Compared with Con group, ${ }^{* * * *} P<0.001$.

significantly reduce the autophagy level of TNBC resistant cells $(p<$ 0.001 ). MDC staining results (Figure $2 \mathrm{C} \& \mathrm{D}$ ) showed that the green fluorescence intensity of drug-resistant strains in the combination group of CCI and DOC was weakened and the autophagosomes was significantly attenuated compared with the control group $(p<0.001)$, indicating that the combination of CCI and DOC could inhibit the autophagy of MDA-MB-231/Doc and MDA-MB-468/Doc, and had a more significant inhibitory effect than CCI or Doc alone. The results of immunofluorescence test (Figure 2E\&F) showed that the fluorescence activity and autophagy related protein LC3 levels of the drug-resistant strains in Doc + CCI, CCI and Doc were lower than those in the control group, and the fluorescence intensity and autophagy related protein levels of the drug-resistant strains in Doc + CCI group were stronger than those in the single application group of Doc and CCI $(p<0.001)$. It was suggested that the combination of drugs can enhance the sensitivity of breast cancer cells to Doc, significantly reduce the expression of LC3, and reduce the level of autophagy.

\section{The role of miR-520d in the reversal of TNBC resistance by CCI}

In this study, MTT method was applied to detect the role of miR$520 \mathrm{~d}$ in the reversal of TNBC resistance by CCI (Figure 3A). Through the MTT assay, it was observed that in MDA-MB-231/Doc and MDAMB-468/Doc strains, the cell viability of drug-resistant strains decreased markedly compared with control group. On the contrary, if the inhibitor of miR-520d was used to inhibit the level of miR-520d, the cell viability of drug-resistant strains improved than that CCI group without inhibitor. The results of MDC staining showed that the autophagy level of CCI group was lower than that of the control group, and the autophagy level of CCI + inhibitor group was higher $(p<0.001)$ (Figure $3 \mathrm{~B} \& \mathrm{C})$. The results of immunofluorescence also showed that inhibition of miR-520d increased the number of autophagic bodies (Figure 3D). It was suggested that the down-regulation of miR-520d can increase the autophagy ability of drug-resistant cells. The results of LC3 showed that the autophagy related protein LC3 in the low expression miR-520d group was higher than that in the control group $(p<0.001)$ (Figure $3 \mathrm{~F}$ ). The above results showed that the down-regulation of miR-520d could inhibit the autophagy of TNBC resistant cells and significantly reduce the sensitivity of TNBC resistant cells promoted by CCI to Doc. 

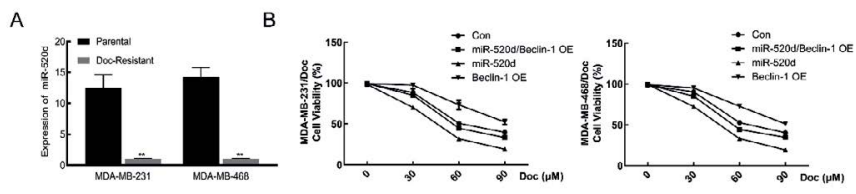

C
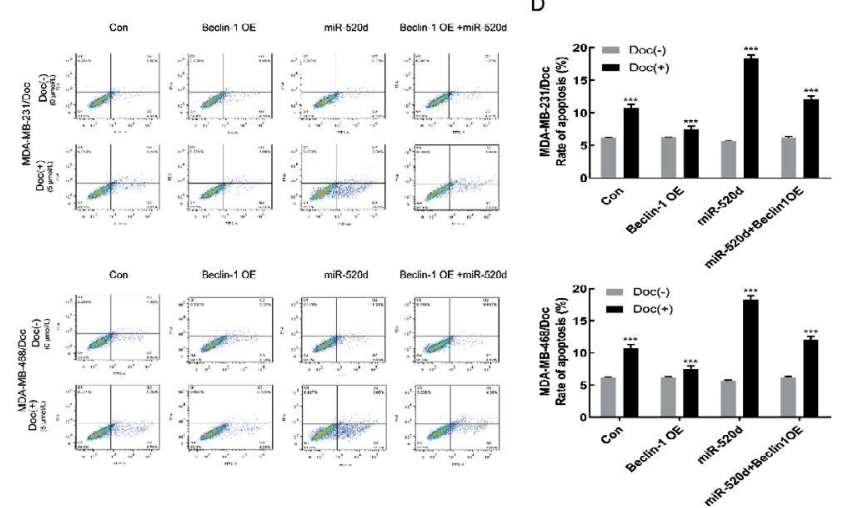

Figure 4A: Detection of miR-520d expression in TNBC by real-time PCR; B: MTT detection the effect of miR-520d on apoptosis under Doc; C: Flow cytometry detection difference of miR-520d and Beclin1 overexpression of under Doc in drug-resistant cell strain. D: Statistics; Compared with Con, ${ }^{* * * *} P<0.001$.

Detection difference of miR-520d and Beclin1 overexpression of under Doc in drug-resistant cell strain

RT-PCR was used to detect the expression level of miR-520d in drug-resistant and non-resistant cells of TNBC cells. The expression level of miR-520d in MDA-MB-231/Doc and MDA-MB-468/Doc was significantly $(p<0.01)$ lower than that of MDA-MB-231 and MDAMB-468, indicating that the markedly low expression level of miR-520d in drug-resistant strains than in the non-resistant cells (Figure 4A). This may suggest that drug resistance in tumor cells was associated with low expression of miR-520d. Through the MTT assay, it was observed that in the two drug-resistant strains MDA-MB-231/Doc and MDAMB-468/Doc, compared with the control group, the cell viability of resistant strains decreased after miR-520d overexpression. Compared with the control group, the Beclin1-OE group increased the viability of drug-resistant cells. When Beclin-1 and miR-520d were simultaneously up-regulated, the viability of drug-resistant cells increased compared with the miR-520d overexpression group. Therefore, the overexpression of miR-520d was posited to reduce the viability of MDA-MB-231/Doc and MDA-MB-468/Doc cells (Figure 4B). On the other hand, it might mean that an increase in the miR-520d expression level could reverse the resistance of TNBC resistant cells to Doc. Under the effect of Doc, the apoptosis rate of each group increased, compared with the groups without Doc effect $(p<0.001)$. In addition, the miR-520d overexpression group had the highest apoptosis rate. Compared with the miR-520d overexpression group, the apoptosis rate decreased slightly after the simultaneous up-regulation of miR-520d and Beclin1 (Figure 4C\&D).

Western blotting detection of the effect of miR-520d on the expression of apoptosis and autophagy-related proteins

Western blotting analysis was applied to detect the expression levels of autophagy-related proteins after overexpression of Beclin 1 and miR-520d. In comparison with the parental strain, the expression of autophagy related protein in the resistant strain was increased, while the expression of p62 was decreased (Figure 5A\&B). Beclin 1 and LC3 levels markedly $(p<0.001)$ decreased after miR-520d overexpression compared with the control group. This finding suggests that up-

regulation of miR-520d reduced the autophagy level of drug-resistant cells. Besides, when Beclin1 and miR-520d were simultaneously upregulated, Beclin1 and LC3 levels were also increased significantly in comparison with the control group $(p<0.001)$ (Figure 5C\&D), which indicates that miR-520d may regulate the autophagy level of drugresistant cells through Beclin1.

\section{Detection of autophagy via MDC staining and immunofluorescenc}

The results from MDC staining (Figure 6A\&B) showed that comparable to the control group, the autophagosomes in the Beclin1 overexpression group (Beclin1-OE) were significantly ( $p$ $<0.001$ ) enhanced, suggesting that Beclin1 overexpression could enhance the autophagic ability of drug-resistant cells. Meanwhile, the autophagosomes in the miR-520d overexpression (miR-520d mimics) group were reduced, which suggest that overexpression of miR-520d could inhibit the autophagic capability of drug-resistant cells. Furthermore, when both Beclin 1 and miR-520d were upregulated simultaneously, the autophagic ability had a certain degree of increment in comparison with the control group $(p<0.001)$. This observation indicated that miR-520d could regulate the autophagy level in drug-resistant cells via Beclin1. Immunofluorescence assay (Figure 6C\&D) showed that autophagy-associated protein, LC3 was inhibited substantially $(p<0.001)$ in cells overexpressing miR-520d compared with the control group while the autophagy level of the cells

A

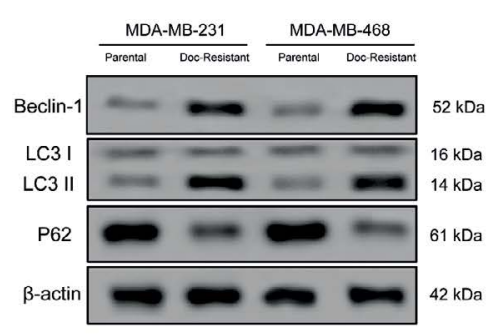

C
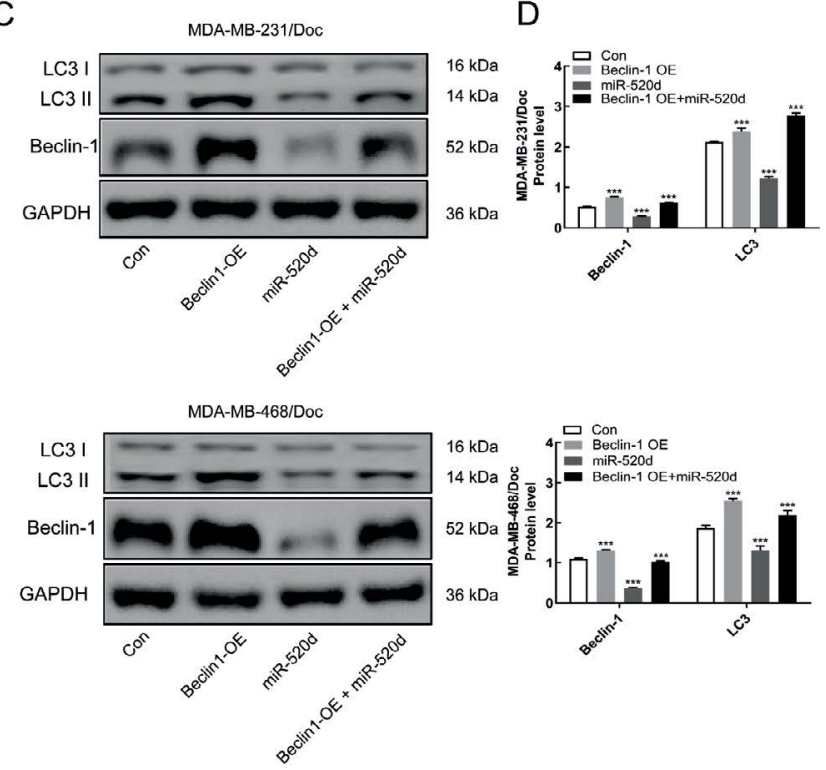

Figure 5A: Western blotting detection the expression level of autophagy-related proteins. B: Statistics. C: Western blotting detection the effect of miR-520d on the expression of autophagy-related proteins. D: Statistics; Compared with Con, ${ }^{* * * *} P<0.001$. 


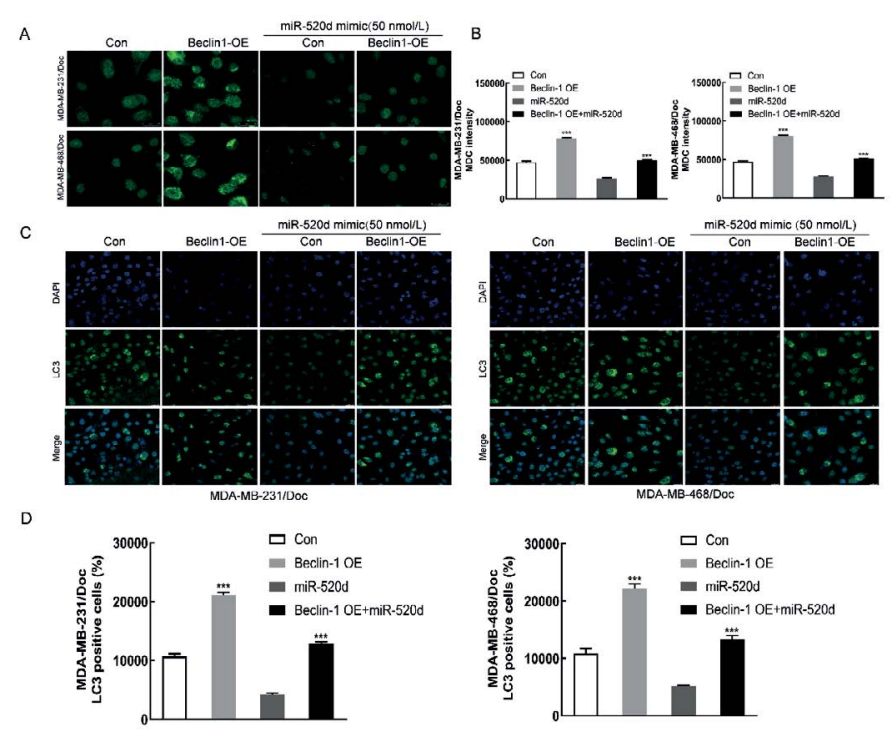

Figure 6A: MDC staining detection the autophagosome formation (Con: control group, Beclin1-OE: Beclin1 Overexpression group, miR-520d mimic/Con: miR-520d overexpression control group, miR-520d mimic/ Beclin1-OE: miR-520d and Beclin1 simultaneous up-regulation group). B: Statistics. C: Immunofluorescence detection the effect of miR-520d on autophagy-associated protein LC3. D: Statistics; Compared with Con, ${ }^{* * * *} P<0.001$

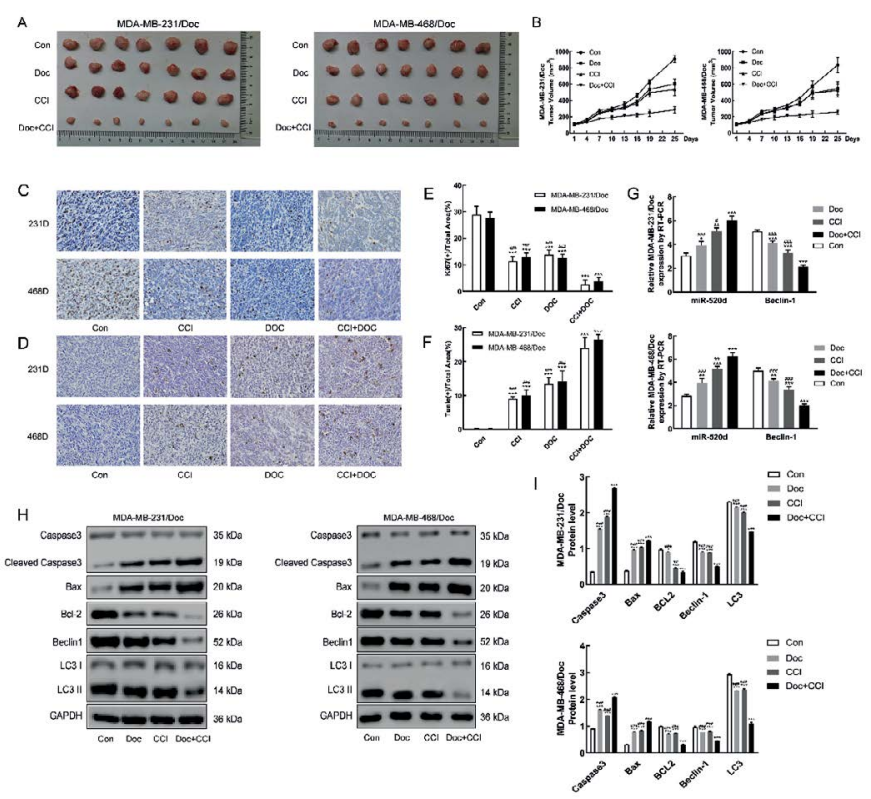

Figure 7: Inhibitory effect of CCI $(15.06 \mathrm{~g} / \mathrm{kg})$, Doc $(10 \mathrm{mg} / \mathrm{kg})$ and their combination on animal tumor. (A: Tumor size comparison of each group; B: Change of tumor volume over time; C: KI67 detects proliferation; D: Tunle detects apoptosis; E\&F: Statistics; G: Detection of miR-520d and Beclin-1 in two drug resistant animal models by RT-PCR; H Detection of apoptosis and autophagy related proteins in two drug resistant animal model by western blotting; I: Statistics; Compared with Con, ${ }^{*} P<0.05,{ }^{* * *} P<0.01,{ }^{* * *} P<0.001$; Compared with Doc + CCI, ${ }^{\# P} P<0.05,{ }^{\# P} P<0.01,{ }^{\# \# P} P<0.001$.)

was decreased. Further experiments showed that overexpression of miR-520d could inhibit the autophagic ability of TNBC resistant cells. Furthermore, Beclin1 overexpression or Beclin1 and miR-520d were simultaneously up-regulated, while autophagy-related protein, LC3 in drug-resistant cells was significantly $(p<0.001)$ increased comparable to the control group.

\section{Animal experiments}

The results of animal experiments showed that the tumor volume of nude mice in Doc + CCI group decreased significantly compared with the control group, which indicated that CCI could enhance the sensitivity of TNBC cells to Doc and enhance the inhibition of tumor cells (Figure 7A\&B). Immunohistochemistry showed that Doc and CCI could promote apoptosis and down-regulate the expression of Ki67 compared with the control group $(p<0.001)$ (Figure 7C\&D). Western blotting was used to detect the effects of Doc, CCI and their combination on apoptosis and autophagy related proteins (Figure $7 \mathrm{H}$ ). The results showed that Doc and CCI could significantly up-regulate the expression of apoptosis protein caspase 3 and $\mathrm{Bax}$, down-regulate the expression of anti-apoptosis protein BCL-2, autophagy related protein LC3 and Beclin-1 $(p<0.001)$. RT-PCR showed that CCI could up-regulate the expression of miR-520d and down-regulate the expression of Beclin-1 compared with the control group $(p<0.05)$. However, when Doc and CCI were jointly applied to the two drug resistant animal models (MDA-MB-231/Doc and MDA-MB-468/ Doc), it could significantly up-regulate the expression of miR-520d and down-regulate the expression of Beclin-1 $(p<0.001)$ (Figure 7G).

\section{Discussion}

Actually, TCM has been shown to exhibit unique advantages in the differentiation and treatment of breast cancer, which can significantly improve the quality of life and prolong the survival period of patients [29]. According to the TCM, breast cancer is caused by emotional disorder, stagnation of liver Qi, blockage of meridians and collaterals, block of Qi mechanism, endogenesis of phlegm and blood stasis, accumulation of Qi and blood for a long period of time [30]. Although, the pathogenesis of breast cancer is complicated, phlegm and blood stasis blocking collaterals, heat and toxin are regarded as the main cause of the disease. Weakness of vital energy is the basis of recurrence and metastasis of breast cancer, while the key factor for recurrence and metastasis of the disease is toxic phlegm and stasis. Therefore, therapy for TNBC is based on the theory of toxin deficiency, phlegm and blood stasis, which is regarded as a treatment method of TCM to prevent recurrence and metastasis [31]. The treatment of TCM has important clinical significance for the postoperative complications of breast cancer and the alleviation of the side effects of radiotherapy and chemotherapy. Among these treatment options is CCI, which is a commonly used as adjuvant of chemotherapy for TNBC. Notably, CCI is composed of the extracts from Cantharidin, Radix Ginseng, Astragaloside and Eleutherococcus senticosus. Usually, it is known as a pure TCM preparation of modern formula. In the prescription, cantharidin with the main function as evil expeller is the monarch drug. Cantharidin is shown to contain cantharidin, which is the main active component with antitumor activity. Likewise, radix ginseng and astragaloside are the other main drugs in the prescription. In order to enhance the strengthening effect of these two drugs, eleutherococcus senticosus is usually used as an "adaptogen" like radix ginseng. Functionally, the medicine has the ability to break blood and remove blood stasis, clear away heat and toxin, attack toxin and erode sore, strengthen the body, improve immunity, activate tumor suppressor genes, kill cancer cells, and have synergistic as well as auxiliary effects on chemotherapy. The main component of CCI, cantharidin, has the effect of directly killing tumor cells. The anti-tumor active component of cantharidin extract is the norcantharidin, which has the inhibitory effect on breast cancer cells [32]. Besides, it has the characteristics of 
anticancer without myelosuppression, and can resist the decline of blood cells in radiotherapy and chemotherapy. Clinical observation has shown that the compound cantharis has certain curative effect on TNBC and can effectively prevent postoperative recurrence and metastasis [33]. Modern medical research has confirmed that CCI can play a therapeutic role in malignant tumors through multiple channels and multiple targets. These include interfering with DNA and RNA biosynthesis of tumor cells, killing tumor cells directly, inhibiting the expression of oncogene, inducing apoptosis of tumor cells, inhibiting tumor angiogenesis, reversing multidrug resistance, protecting bone marrow, regulating immunity, enhancing the activity of lymphokine activated killer cells and natural killer cells [34]. Notwithstanding, the reversal of drug resistance is regarded as one of the mechanisms of antitumor action of compound cantharides. This study therefore further clarified the specific mechanism of the reversal of drug resistance.

The process of autophagy is mainly regulated by autophagy related genes. Beclin-l is a marker protein which activates or initiates autophagy. It is involved in the formation and maturation of autophagy by binding with different autophagy related proteins to form complexes. During autophagy, the expression level of Beclin-1 tends to increase [35]. Beclin-1 can participate in drug resistance of tumor cells by regulating autophagy. Physiologically, Doc was found to induce endoplasmic reticulum stress, which could contribute to the phosphorylation of Bcl-2 mediated by c-Jun amino terminal kinase- 1 , and consequently result in the release of Beclin1 to promote autophagy. Besides, in combination with Bax, the released Beclin-1 could inhibit apoptosis, so that the tumor cells can resist apoptosis, and finally culminate in tumor drug resistance [36]. It has been confirmed that the reduction of Beclin-1 expression level in breast cancer cells could improve its chemosensitivity [37]. Therefore, it is of great significance to investigate the mechanism underlying Beclin-1 expression and regulation of the chemotherapy resistance in breast cancer.

In the current study, a TNBC resistant cell strain with Doc resistance was employed to detect the effects of CCI, Doc and their combination on proliferation and apoptosis of TNBC resistant cell strains. The results showed that the proliferation of TNBC resistant cell strain was significantly inhibited by the combination of drugs, while the apoptosis increased significantly compared with the control group. Moreover, autophagy level decreased in TNBC resistant cell strain of combination of Doc and CCI, and the expression of miR-520 and Beclin-1 downregulated. It was suggested that CCI may reverse the drug resistance by inhibiting the over autophagy of TNBC resistant cell strains.

Of late, scientists have shown much interest in the role of miRNA in the regulation of tumor autophagy [38]. It has been established that the regulation of tumor autophagy by miRNAs is via the regulation of the expression level of autophagy related genes after transcription. For instance, exocrine miRNA-126 can disrupt insulin receptor substrate (IRS)/Glut-4 signal transduction, activate AMP-activated protein kinase (AMPK)/autophagy pathway and stabilize hypoxia -inducible factor 1-alpha (HIF-1a) expression in adipocytes to be produced. Also, the inhibition of miRNA-126 in vivo could reduce tumor growth induced by the adipocytes [39]. In makes caterpillars floppy-7 (MCF-7) breast cell line, miR-486-5p inhibitor induced autophagy and enhanced the autophagy process inducted by AdoMet through increase in PTEN expression and inhibition of AKT signaling [40]. Meanwhile, some studies have shown that miR-224-5p could inhibit autophagy by targeting Smad4 in MDA-MB-231 cells, while miR-224-5p/Smad4 autophagy signal axis may act as a new target for breast cancer treatment [41]. In addition, in TNBC, the autophagy defect mediated by miR-20a may be regarded as a new mechanism of the carcinogenic function of miRNA in the process of breast cancer [42]. Besides, miR-376a was shown to inhibit autophagy induced by starvation in the MCF-7 cells and Huh-7 cells via the inhibition of atg $4 \mathrm{~b}$ and Beclin-1 expression [43]. Further literature search showed that miRNA could affect the drug resistance of tumor cells by regulating the autophagy level of cells. Exemplary, miR181 could not only inhibit the autophagy level of breast cancer MCF-7 cells, but also inhibit the autophagy induced by drugs such as etoposide and rapamycin, as well as further reversing the drug resistance [44]. In breast cancer, miR-214 could enhance the sensitivity of the breast cancer cells to tamoxifen via autophagy inhibition [45]. Again, miR$129-5 p$ with autophagy regulatory function could down regulate the expression of Beclin-1 gene by combining with 3' UTR sequence, and subsequently play its role in inhibiting autophagy of a variety of tumor cells, namely cancer of the breast, lung and other cells [46].

In order to further explore the molecular mechanism of CCI in the reversal of the drug resistance of TNBC, we used miRNA chip to detect the drug-resistant cells of TNBC before and after the treatment with CCI. It was found that compound cantharidin could significantly increase the level of miR-520d. In addition, RT PCR also affirmed this result. Presently, miR-520d was observed to act as a possible specific biomarker for the breast cancer diagnosis, because it is expressed highly in cancer of the breast, especially in the breast cancer patients with negative HER-2 [47]. In this regard, the relationship between miR-520d and tumor has attracted more attention by scientists [48]. Previously, miR-520d has been reported to inhibit the proliferation, migration and invasion of gastric cancer cells through down regulation of EphA2 expression [49]. Also, it has been observed earlier that in the MDA-MB-231, over expression of miR-520d could reduce cell proliferation and migration [50]. In view of the existing literature, we affirmed that miR-520d could bind to the 3' UTR region of Beclin-1 mRNA, and significantly inhibit the expression of Beclin-1 protein in TNBC resistant cells. Besides, the results showed that miR-520d mimic could significantly increase the sensitivity of Doc to the resistant cells. Contrariwise, miR-520d inhibitor significantly inhibited the reversal effect of CCI on the drug resistance of TNBC cells. In addition, the effect of CCI on TNBC resistant animal model through miR-520d/ Beclin-1 signal axis was verified by animal experiments in this study. Altogether, these findings indicate that the miR-520d/Beclin-1 signal axis is the main mechanism of CCI in reversing the chemotherapy resistance of TNBC.

\section{Conclusion}

This study showed that CCI could inhibit the high autophagy level of the drug-resistant cells of the TNBC by up-regulating the expression of miR-520d and targeting Beclin-1. This could increase the drug sensitivity of the drug-resistant cells of TNBC to Doc and reverse the drug resistance of the TNBC. This study will aid in clarifying the internal relationship among compound cantharidin, autophagy and drug resistance, as well as improve the mechanism of chemotherapy resistance of TNBC. Thus, this study provides experimental basis for clinical treatment of TNBC using compound cantharidin as an auxiliary combination.

\section{Author contributions}

Xia Sheng, Dianxu Feng and Ke Xu contributed to conceiving the research. Hongchang $\mathrm{Li}$ and Huan Liu conducted the in vitro experiment, analyzed the data, and drafted the manuscript. Yafeng Chen and Jie Li conducted animal experiments. Xia Sheng, Dianxu 
Feng and Ke Xu critically revised the manuscript. All authors read and approved the final manuscript.

\section{Declaration of Competing Interest}

The authors declare that the research was conducted in the absence of any commercial or financial relationships that could be construed as a potential conflict of interest.

\section{Acknowledgement}

This work was supported by the National Natural Science Foundation of China, Compound cantharis reverses chemotherapy resistance of TNBC via miR-520d/Beclin1 signal axis (No.81703881) and High level key specialist doctors under the medical teaching research collaborative health service system in Minhang District (No. 2020MZYS02).

\section{References}

1. Bartsch R, Ziebermayr R, Zielinski CC, Steger GG (2010) Triple-negative breast cancer. Wien Med Wochenschr 160: 174-181. [Crossref]

2. Yao H, He G, Yan S, Chen C, Song L, et al. (2017) Triple-negative breast cancer: is there a treatment on the horizon? Oncotarget 8: 1913-1924. [Crossref]

3. Podo F, Buydens LM, Degani H, Hilhorst R, Klipp E, et al. (2010) Triple-negative breast cancer: Present challenges and new perspectives. MOL ONCOL 4: 209-229. [Crossref]

4. Jin J, Zhang W, Ji W, Yang F, Guan X (2017) Predictive biomarkers for triple negative breast cancer treated with platinum-based chemotherapy. Cancer Biol Ther 18: 369378. [Crossref]

5. Wu ZH, Lin C, Liu MM, Zhang J, Tao ZH, et al. (2016) Src Inhibition Can Synergize with Gemcitabine and Reverse Resistance in Triple Negative Breast Cancer Cells via the AKT/c-Jun Pathway. PLoS One 11: e0169230. [Crossref]

6. Afghahi A, Timms KM, Vinayak S, Jensen KC, Kurian AW, et al. (2017) Tumor BRCA1 Reversion Mutation Arising During Neoadjuvant Platinum-Based Chemotherapy in Triple-Negative Breast Cancer Is Associated with Therapy Resistance. Clin Cancer Res 23: 3365-3370. [Crossref]

7. Ishii Y, Fujimoto S, Okazaki K, Miyoshi M, Furihata T, et al. (2011) Fractionated administration of carboplatin/paclitaxel reduces neurotoxicity in patients with advanced non-small cell lung cancer. Anticancer Drugs 22: 926-32. [Crossref]

8. Guan X, Gu S, Yuan M, Zheng X, Wu J (2019) MicroRNA-33a-5p overexpression sensitizes triple-negative breast cancer to doxorubicin by inhibiting eIF5A2 and epithelial-mesenchymal transition. Oncol Lett 18: 5986-5994. [Crossref]

9. Battista RA, Resnati M, Facchi C, Ruggieri E, Cremasco F, et al. (2018) Autophagy mediates epithelial cancer chemoresistance by reducing p62/SQSTM1 accumulation. PLoS One 13: e0201621. [Crossref]

10. Das CK, Linder B, Bonn F, Rothweiler F, Dikic I, et al. (2018) BAG3 Overexpression and Cytoprotective Autophagy Mediate Apoptosis Resistance in Chemoresistant Breast Cancer Cells. Neoplasia 20: 263-279. [Crossref]

11. Kim JS, Park SY, Lee SA, Park MG, Yu SK, et al. (2014) MicroRNA-205 suppresses the oral carcinoma oncogenic activity via down-regulation of Axin-2 in KB human oral cancer cell. Mol Cell Biochem 387: 71-79. [Crossref]

12. Zhang G, Li N, Li Z, Zhu Q, Li F, et al. (2015) MicroRNA-4717 differentially interacts with its polymorphic target in the PD1 3' untranslated region: A mechanism for regulatingPD-1 expression and function in $\mathrm{HBV}$-associated liver diseases. Oncotarget 6: 18933-18944. [Crossref]

13. Chen J, Tian W, Cai H, He H, Deng Y (2012) Down-regulation of microRNA-200c is associated with drug resistance in human breast cancer. Med Oncol 29: 2527-2534. [Crossref]

14. Pan B, Yi J, Song H (2013) MicroRNA-Mediated Autophagic Signaling Networks and Cancer Chemoresistance. Cancer Biother Radiopharm 28: 573-578. [Crossref]

15. Ambros V (2003) MicroRNA Pathways in Flies and Worms: Growth, Death, Fat,Stress, and Timing. Cell 113: 673-676. [Crossref]

16. Carrington JC, Ambros V, et al. (2003) Role of MicroRNAs in Plant and Animal Development. Science 301: 336-338. [Crossref]
17. Sempere LF, Sokol NS, Dubrovsky EB, Berger EM, Ambros V (2003) Temporal regulation of microRNA expression in Drosophila melanogaster mediated by hormonal signals and Broad-Complex gene activity. Dev Biol 259: 9-18. [Crossref]

18. He L, Hannon GJ (2004) MicroRNAs: small RNAs with a big role in gene regulation. Nat Rev Genet 5: 522-531. [Crossref]

19. Yu AM, Pan YZ (2012) Noncoding microRNAs: small RNAs play a big role in regulation of ADME? Acta Pharm Sin B 2: 93-101. [Crossref]

20. Chen S, Jiao JW, Sun KX, Zong ZH, Zhao Y (2015) MicroRNA-133b targets glutathione S-transferase $\pi$ expression to increase ovarian cancer cell sensitivity to chemotherapy drugs. Drug Des Devel Ther 9:5225-5235. [Crossref]

21. Huang S, Qi P, Zhang T, Li F, He X (2019) The HIF- $1 \alpha /$ miR-224-3p/ATG5 axis affects cell mobility and chemosensitivity by regulating hypoxia-induced protective autophagy in glioblastoma and astrocytoma, Oncol Rep 41: 1759-1768. [Crossref]

22. Galluzzi L, Pietrocola F, Levine B, Kroemer G (2014) Metabolic Control of Autophagy. Cell 159: 1263-1276. [Crossref]

23. Kubisch J, Türei D, Földvári-Nagy L, Dunai ZA, Zsákai L, et al. (2013) Complex regulation of autophagy incancer - Integrated approaches to discover the networks that hold a double-edged sword. Semin Cancer Biol 23: 252-261. [Crossref]

24. Zhu ZY, Xue JX, Yu LX, Bian WH, Zhang YF, et al. (2018) Reducing postsurgica exudate in breast cancer patients by using San Huang decoction to ameliorate inflammatory status: a prospective clinical trial. Curr Oncol 25: e507-e515. [Crossref]

25. Li HC, Xia ZH, Chen YF, Yang F, Feng W, et al. (2017) Cantharidin Inhibits the Growth of Triple-Negative Breast Cancer Cells by Suppressing Autophagy and Inducing Apoptosis in Vitro and in Vivo. Cell Physiol Biochem 43: 1829-1840. [Crossref]

26. Xiao Z, Jiang Y, Chen XF, Wang CQ, Xu WH, et al. (2020) The hepatorenal toxicity and tumor response of chemotherapy with or without aidi injection in advanced lung cancer: A meta-analysis of 80 randomized controlled trials. Clin Ther 42: 515-543. [Crossref]

27. Li HC, Liu WY, Wang JF, et al. (2020) Compound Cantharis Reverses Chemotherapy Resistance of TNBC via MiR-520d/Beclin1 Signal Axis. Modernization of Traditional Chinese Medicine and Materia Medica-World Science and Technology. 22: 970-977.

28. Liu H, Li HC, Chen YF, et al. (2019) miR-520d over-expression reverses chemotherapy resistance of TNBC via inhibiting autophagy protein Beclin1. Chin J Cancer Biother 10: 1062-1067. [Crossref]

29. McPherson L, Cochrane S, Zhu X (2016) Current Usage of Traditional Chinese Medicine in the Management of Breast Cancer: A Practitioner's Perspective. Integr Cancer Ther 15: 335-342. [Crossref]

30. Dong MX, Chang P (2019) Clinicopathological characteristics and TCM differentiation analysis of triple negative breast cancer. China's Naturopathy 27: 71-73. [Crossref]

31. Li KX, Zhou M (2015) Research Progress of Triple Negative Breast Cancer and Traditional Chinese Medicine Treatment. Hubei Journal of Traditional Chinese Medicine 37: 76-78. [Crossref]

32. Huan SK, Wang KT, Yeh SD, Lee CJ, Lin LC, et al. (2012) Scutellaria baicalensis alleviates cantharidin-induced rat hemorrhagic cystitis through inhibition of cyclooxygenase-2 overexpression. Molecules 17: 6277-6289. [Crossref]

33. B Yan, H Li (2013) Clinical observation of the effect of compound cantharis capsule combined with chemotherapy in the treatment of metastatic colorectal cancer. 25: 1017 1019. [Crossref]

34. Chu LW, Wen ZY, Zhang LG (2014) The effect of Aidi Injection on the peripheral blood lymphocytes of breast cancer patients. China practical medicine 9: 27-28. [Crossref]

35. Yang XQ, Zhang YL (2014) Characterization of glutathione S-transferases from Sus scrofa, Cydia pomonella and Triticum aestivum: their responses to cantharidin. Enzyme Microb Technol 69: 1-9. [Crossref]

36. Tai WT, Shiau CW, Chen HL, Liu CY, Lin CS, et al. (2013) Mcl-1-dependent activation of Beclin-1 mediates autophagic cell death induced by sorafenib and SC-59 in hepatocellular carcinoma cells. Cell Death Dis 4: e485. [Crossref]

37. Gu Y, Chen T, Li G, Xu C, Xu Z, et al. (2016) Lower Beclin-1 downregulates HER2 expression to enhance tamoxifen sensitivity and predicts a favorable outcome for ER positive breast cancer. Oncotarget 8: 52156-52177. [Crossref]

38. Subramaniam S, Jeet V, Clements JA, Gunter JH, Batra J, et al. (2019) Emergence of MicroRNAs as Key Players in Cancer Cell Metabolism. Clin Chem 65: 1090-1101. [Crossref] 
39. Wu Q, Li J, Li Z, Sun S, Zhu S, et al. (2019) Exosomes from the tumour-adipocyte interplay stimulate beige/brown differentiation and reprogram metabolism in stromal adipocytes to promote tumour progression. J Exp Clin Cancer Res 38: 223. [Crossref]

40. Ilisso CP, Delle Cave D, Mosca L, Pagano M, Coppola A, et al. (2018) S-Adenosylmethionine regulates apoptosis and autophagy in MCF-7 breast cancer cells through the modulation of specificmicroRNAs. Cancer Cell Int 18: 197. [Crossref]

41. Cheng Y, Li Z, Xie J, Wang P, Zhu J, et al. (2018) MiRNA-224-5p inhibits autophagy in breast cancercells via targeting Smad4. Biochem Biophys Res Commun 506: 793798. [Crossref]

42. Liu L, He J, Wei X, Wan G, Lao Y, et al. (2017) MicroRNA-20a-mediated loss of autophagy contributes to breast tumorigenesis by promoting genomic damage and instability. Oncogene 36: 5874-5884. [Crossref]

43. Bortnik S, Choutka C, Horlings HM, Leung S, Baker JH, et al. (2016) Identification of breast cancer cell subtypes sensitive to ATG4B inhibition. Oncotarget 7: 66970-66988. [Crossref]

44. Tekirdag KA, Korkmaz G, Ozturk DG, Agami R, Gozuacik D, et al. MIR181A regulates starvation- and rapamycin-induced autophagy through targeting of ATG5. Autophagy 2013 9: 374-385. [Crossref]
45. Yu X, Luo A, Liu Y, Wang S, Li Y, et al. (2015) MiR-214 increases the sensitivity of breast cancer cells to tamoxifen and fulvestrant through inhibition of autophagy. Mol Cancer 14: 208. [Crossref]

46. Duan L, Hao X, Liu Z, Zhang Y, Zhang G, et al. (2014) MiR-129-5p is down-regulated and involved in the growth, apoptosis and migration of medullary thyroid carcinoma cells throughtargeting RET. FEBS Lett 588: 1644-1651. [Crossref]

47. Savad Sh, Mehdipor P, Shirdast H, et al. (2013) MiR-520d expression analysis in breast cancer. Tehran University Medical Journal 71: 90-95. [Crossref]

48. Danese E, Minicozzi AM, Benati M, et al. (2017) Reference miRNAs for colorecta cancer: analysis and verification of current data. Sci Rep 7(1): 8413. [Crossref]

49. Li R, Yuan W, Mei W, Yang K, Chen Z (2014) MicroRNA 520d-3p inhibits gastric cancer cell proliferation, migration, and invasion by downregulating EphA2 expression. MolCell Biochem 396: 295-305. [Crossref]

50. Tsukerman P, Yamin R, Seidel E, Khawaled S, Schmiedel D, et al. (2014) MiR-520d-5p directly targets TWIST1 and downregulates the metastamiR miR-10b. Oncotarget 5 : 12141-12150. [Crossref]

Copyright: (C2021 Li H. This is an open-access article distributed under the terms of the Creative Commons Attribution License, which permits unrestricted use, distribution, and reproduction in any medium, provided the original author and source are credited. 